\title{
Detection and Quantification of Anti-Inflammatory Cytokine in Sera and Urine of Sudanese Patients Infected with Schistosoma haematobium
}

\author{
A. Hammad1, Manahil Nouri'2, Abdelbagi Alfadil ${ }^{3 *}$, \\ H. A. Musa4, M. A. Osman ${ }^{5}$, A. Bashir ${ }^{6}$, Nawal Eltayeb Omer ${ }^{7}$, \\ Yasir Hassan $^{8}$, A. Alfarazdeg 9 , A. Mustafa ${ }^{10}$ \\ ${ }^{1}$ Department of Parasitology, National Ribat University, Khartoum, Sudan \\ ${ }^{2}$ Department of Community Medicine, Al Neelain University, Khartoum, Sudan \\ ${ }^{3}$ Department of Immunology, National Ribat University, Khartoum, Sudan \\ ${ }^{4}$ Department of Microbiology, National Ribat University, Khartoum, Sudan \\ ${ }^{5}$ Department of Microbiology, University of Bacht Elruda, Eldeum, Sudan \\ ${ }^{6}$ Department of Microbiology, Khartoum University, Khartoum, Sudan \\ ${ }^{7}$ Department of Pathology, Khartoum College of Medical Sciences, Khartoum, Sudan \\ ${ }^{8}$ Sudan Medical Specialization Board, Khartoum, Sudan \\ ${ }^{9}$ Department of Biochemistry, Khartoum University, Khartoum, Sudan \\ ${ }^{10}$ Soba Hospital, Khartoum University, Khartoum, Sudan \\ Email: "elfadilsss@yahoo.com
}

Received 26 October 2015; accepted 20 March 2016; published 23 March 2016

Copyright (C) 2016 by authors and Scientific Research Publishing Inc.

This work is licensed under the Creative Commons Attribution International License (CC BY).

http://creativecommons.org/licenses/by/4.0/

(c) (i)

\section{Abstract}

Cytokines have been in focus of scientific in for more than decade now. Analyzing their expression has enabled a better understanding of pathogenesis of various diseases. Moreover, they are now for beyond their stage when they were of interest for pathophysiological research. In the present study, we used an assay to anti-inflammatory cytokines. One hundred and thirty sera and urine from egg positive individuals and 42 samples of healthy individuals in Eldeum locality-an endemic area of $S$. haematobum and 28 healthy individuals from non-endemic area were tested. Concentration of IL5 and IL10 is reverse. IL5 is low in serum but high in urine before treatment and high in serum and low in urine after treatment. IL10 is high in serum, low in urine before treatment and low in serum, high in urine after treatment.

"Corresponding author.

How to cite this paper: Hammad, A., et al. (2016) Detection and Quantification of Anti-Inflammatory Cytokine in Sera and Urine of Sudanese Patients Infected with Schistosoma haematobium. Open Journal of Medical Microbiology, 6, 32-41.

http://dx.doi.org/10.4236/ojmm.2016.61006 


\section{Keywords}

\section{Schistosoma haematobum, Interleukin, ELISA}

\section{Introduction}

Schistosomiasis is one of the wide spreads of all parasitic infection of man. The [1], estimated that Schistosomiasis and soil transmitted helminthes represent more than $40 \%$ of the global disease burden caused by all tropical diseases excluding malaria. Urinary Schistosomias caused by Schistosoma haematobium is endemic in 53 countries in the Middle East and most of the African continent [2], especially in the rural areas where only the surface water bodies are sources of water supply [3], Schistosoma haematobium infects over 112 million individuals and results in 150,000 deaths annually in sub-Saharan Africa [4]. In a study from Kenya, individuals with chronic infections and hepatosplenic disease appear to have predominantly Th1 responses to Schistosoma antigens and more severe pathology [5] [6] in his study from Schistosoma-endemic area in Zimbabwe showed that egg-positive people had significantly higher levels of specific antibodies, IL-2, IFN- $\gamma$ and IL-23. In contrast, egg-negative individuals had significantly higher circulating IL-10, IL-4, IL-13, and IL-21 that were detected with high frequency in all participants. When he correlated cytokines to age, he found that IL-4 and IL-10 increased significantly as did Schistosoma-specific antibodies. When age was combined with the infection status, IL-5 declined over time in egg-positive, while increased with age in the egg-negative group. Experimental study done on mouse showed that Th1 responses were predominate in early acute phase, followed by the emergence of Th2 responses (stimulated by egg antigens), and decreased in Th1 responses (down-modulated through an IL10-dependent mechanism). [7] [8] studied the cytokine responses to Schistosoma haematobium in a Zimbabwean population and found that the infection rose to the peak in childhood (11 - 12 years) followed by a sharp decline in infection intensity. The parasite-specific cytokine responses showed that IL-4, IL-5 but not IFN $\gamma$ followed distinct age profiles. He concluded that IL-10 was produced early and peaking in the youngest age group and declining thereafter, while IL-4 and IL-5 responses were developed slowly with later peak. IL-10 was high among egg positive. Also he found that the high IL-10 individuals have little or no IL-5. In vitro stimulation of blood taken from people in low and high prevalence of Schistosoma infection after treatment showed that blood sample from children from low prevalence village produce more IL-4 $(\mathrm{P}<0.001)$. But residence in high prevalence village produce IL-10 (P < 0.006) in response to culture with SEA and IL-5 (P < 0.02) with PHA for 48 hrs. [9] [10] studied the production of IL-4 and IL-5 in vitro stimulation of blood from endemic area of Zimbabwe for 24 or 48 hrs. He found that the sample produce IL-4 or IL-5 but rarely both. But when cultured for 72 hrs, he found that both cytokines were detected. Immune response to IL-5 and IL-10 study done on Schistosoma infected patients treated many times showed high levels of IL-5. After adjusting for variable (sex, age and infection status) at the study onset, high levels of IL-10 were showed to be a risk factor for re-infection and high levels of IL-5 were associated with development of hematuria [11]. In a study using epithelial cells from the human urinary tract, Escherichia coli elicited high levels of IL-6 but not TNF- $\alpha$ whereas human peripheral blood monocytes secreted both IL-6 and TNF- $\alpha$ as well as other cytokines in response to E-coli [12]. A study of local cytokine responses in the urinary tract mucosa may yield important information about their role in the pathogenesis in the affected organs during Schistosoma haematobium infection. The only study that measuring the level of cytokine in urine of Schistosoma haematobium infected individual was done in Kenyans school children [13]. This study aimed to investigate the relationship between serum and urinary cytokines (interleukin- 4 interleukin-5 and interleukin-10 in Schistosoma haematobium infected individual living in two villages in the White Nile state, Sudan.

\subsection{Aim of the Study}

Since World Health Organization (WHO) recommends that infection levels are determined prior to designing and implementing control programs as the treatment regimens. In this study we aim to detect and quantification of Anti-inflammatory cytokines in both sera and urine of patients with urinary schistosomiasis. Before and after treatment. 


\subsection{Ethical Consideration}

Ethical and institutional approval for the study was obtained from the medical research council of the National Ribat University. Permission for initiation of the study in the area was obtained from the Health Services Director-Elduiem locality. Also a meeting was held with the chief of the targeted villages and the objectives and the methods were clearly explained. They were informed that they have the right to be included or to quit at any time. Written informed consent was obtained from eligible individuals and oral consent was obtained from the participant and parents/guardian before sampling.

\section{Materials and Methods}

\subsection{Study Design}

This is a cross-sectional and case control study conducted to study Anti-inflammatory Cytokine in sera and urine of Sudanese patients infected with Schistosoma haematobium.

\subsection{Study Area}

This study was conducted in two villages, Hilat Salim which is located approximately $15 \mathrm{Km}$ south of Elduiemtown in the eastern part of the White Nile bank. Water contact sites (main canal) are of 5 minutes' walk from all houses. The second village is Hilat Saeed which is located $25 \mathrm{Km}$ south Elduiem in western part of the White Nile. Water is collected from a site 10 minutes' walk from the village. The residents have no access to piped water and they depend principally on water from the White Nile and the canal, for domestic and agriculture purposes. The communities of the two villages are mainly peasant farmers who grow maize, wheat and vegetables. Fishing is carried in the White Nile and temporary pools created by the overflow of the White Nile during the rainy season.

These villages were chosen because there is no other helminthes and the prevalence of Schistosoma mansoni is low (personal communication information from team of faculty of medicine university of Becht Elruda), together with different prevalence and intensities of Schistosoma haematobium infection in close proximity. Also the villages are not included in the National schistososme control programmer, meaning that the natural immune responses of the study population could be studied without effect of drugs alteration.

\subsection{Study Population}

A total of two hundred Sudanese individuals from Eldeum locality included in study. One hundred and thirty sera and urine from egg positive individuals and 42 samples of healthy individuals in Eldeum locality-an endemic area of $S$. haematobum and 28 healthy individuals from non-endemic area were tested.

\subsection{Sampling and Procedures}

\subsubsection{Urine and Stool Collection and Examination}

A single terminal urine samples (20 - $50 \mathrm{ml}$ ) was collected in $50 \mathrm{ml}$ containers from each individual of the study population. The samples were obtained between 10:00 a.m. And 14:00 P.m. Few drops of saponin solution were added to the samples with visible hematuria to enhance clarity in microscopy [14]. The specimens were appropriately labelled with identification numbers and placed in cold box with ice packs. They were processed $1-2$ hrs after collection in the field. $10 \mathrm{ml}$ was filtered through a $25 \mathrm{~mm}$ nucleosome filter (12 $\mu$ m pore size) [15]. The filter was examined microscopically for Schistosoma haematobium eggs. The intensity was reported as number of egg/10ml urine. The degree of intensity was categorized as light infection $(\leq 50 \mathrm{ova} / 10 \mathrm{ml}$ of urine $)$ and as heavy infection ( $>50 \mathrm{ova} / 10 \mathrm{ml}$ urine) according to the World Health Organization [16] [17]. To rule out Schistosoma mansoni eggs and other intestinal helminthes, stool specimens were collected from all individual who have Schistosoma haematobium eggs in their urine, and processed following the Kato Katz procedure [18], The urine samples of the participants were aliquoted in cryotubes and stored at $-20^{\circ} \mathrm{C}$.

\subsubsection{Blood Samples}

Five milliliters of venous blood was collected in plain container from each Schistosoma haematobium infected volunteer and allowed to clot at room temperature. The sera were separated using centrifugation at $3000 \mathrm{rpm}$ for 
10 minutes. Then aliquoted in cryotubes and frozen at $-20^{\circ} \mathrm{C}$. Their peripheral blood from all participant was examined for plasmodium parasite using ICT malaria with two species device (P.F \& P.v) (SD Bio standard Diagnostics PVT-LTD India) to exclude malaria infection. All the positive participants were offered anti-helminthic treatment with the recommended dose of praziquantel, $40 \mathrm{mg} / \mathrm{Kg}$ body weight after collection of blood samples. Malaria cases were treated according to the treatment regime prescribed by the Ministry of health in Sudan.

A second blood and urine sample was collected from all the participant four weeks after treatment and processed as before. All the samples were transported frozen in cold box to the laboratory of Parasitology Department in the College of Medical laboratory Sciences National Ribat University, and stored at $-80^{\circ} \mathrm{C}$. The samples were deforested for the first time for prior to testing.

\subsubsection{Control Samples}

A group of 42 individuals were chosen from the area of the study on the basis that they had negative urine samples for Schistosoma haematobium ova and had no past history of Schistosoma. Infection in addition they were neither ill nor under any type of therapy at the time of sample collection. This represent controls from the endemic area. Another group of 28 apparently healthy individuals, living in non-endemic area, were included in the study as controls from a non-endemic area.

\subsubsection{Cytokines Measurement}

Enzyme-Linked Immunosorbent Assay (ELISA) kits e Bioscience were used to determine the levels of three cytokines (IL-5 and IL-10) in serum and urine samples of Schistosoma haematobium infected individuals and controls from the endemic and non-endemic areas. All samples were run in single and the concentrations were calculated using standard curves.

\subsubsection{Assay Procedure}

The assay procedures the same for all three cytokines both in sera and urine. Ninety six polystyrene microtiter plate (corning costar 9018) were coated with recommended concentration of capture antibody $100 \mu \mathrm{l} /$ well in coating buffer. The plate was sealed and incubated overnight at $4^{\circ} \mathrm{C}$. Wells were aspirated (emptied) and washed five times with $250 \mu \mathrm{l} /$ well washing buffer one minute a time were allowed for soaking during each wash step. Plate was blotted on absorbent paper. $200 \mu \mathrm{l}$ of $1 \mathrm{x}$ assay diluents were added/well and the plate was incubated at room temperature for one hour (blocking). Aspiration and wash was done as before. Standards samples were diluted with $1 \mathrm{x}$ assay diluents. Two fold serial dilution of the top standards were performed to make the standard curve, and $100 \mu \mathrm{l} /$ well of standard were added to appropriate wells, also $100 \mu \mathrm{l} /$ well of samples were added to appropriate wells. The plate was sealed and incubated over night at $4^{\circ} \mathrm{C}$. Aspiration/ wash was done as before. $100 \mu \mathrm{l} /$ well of detection antibody diluted in $1 \mathrm{x}$ assay diluents was added to each well, and the plate was sealed and incubated at room temperature for one hour. Aspiration and washing was done as before. Subsequently 100 $\mu \mathrm{l}$ of Avidin-horseradish peroxidase diluted in assay diluents was added to the wells and sealed and incubated at room temperature for 30 minutes. Aspiration/wash was done as before (soaked in wash buffer for 1 - 2 minutes prior to aspiration, repeated for a total of 7 washes. $100 \mu \mathrm{l} /$ well of substrate solution was added to each well and then incubated at room temperature for 15 minute. The reaction was stopped by adding $50 \mu \mathrm{l}$ of stop solution. Optical density of each well was measured with a micro plate reader at $450 \mathrm{~nm}$.

\subsubsection{Statistical Analysis}

The data were analyzed using Statistical Package for Social Sciences (SPSS version, 17). Thus parametric methods were used to evaluate results. The study population was categorized as infected (positive egg) or uninfected (endemic and non-endemic controls). Significance of difference, between mean values were assessed by student's t-test. P-values less than 0.05 were regarded as significant. The comparison between Schistosoma egg positive and egg negative were analyzed by chi-square test. The relation between cytokine levels and infection intensity, as well as between cytokines in pair were conducted using a one-tailed non parametric spearman correlation procedure [19].

\section{Results}

A total of 130 individual met the inclusion criteria of the study, 81 (62\%) males and 49 (38\%) females. Mean 
infection intensity for the study population was 61.92 eggs $/ 10 \mathrm{ml}$ urine (range 0 - 430 eggs $/ 10 \mathrm{ml}$ urine, standard deviation (57.66). The prevalence of Schistosoma haematobium infection, according to different age groups, of 130 volunteers were found among young people aged ( 3 - 13 year old), and then decreased with increasing of age. There were a significant difference in infection prevalence between males $62 \%$ and females $38 \%$. Figure 1 showed the intensity of the infection (53.1\% light infection and $46.9 \%$ heavy infection), according to the WHO classification. The intensity in the majority of participants was considered as light infected.

\section{Cytokine Profile}

The cytokine profile measurement in sera of the infected individual, before and one month after treatment, showed high levels of IL-5 after treatment than before treatment, but the difference was not significant $(\mathrm{P}=$ 0.032) but in case of IL-10 the high production was detected before treatment and the difference was found to be significant $(\mathrm{P}=0.000)$. Figure 2(a) and Figure 2(b) show the mean levels of cytokine profile in serum before and after treatment.

Table 1 summaries the cytokine profile in urine before treatment and one month after treatment. There was high level of different cytokines before treatment than after treatment. The difference was statistically significant in case of IL-5 ( $\mathrm{P}=0.001)$, but the differences were insignificant in case of IL-10 (P = 0.477). In case of IL-5 and IL-10 the detected amount in both sera and urine were found to be reverse, IL-5 low in serum but high in urine before treatment and high in serum and low in urine after treatment. IL-10 high in serum low in urine before treatment and low in serum and high in urine after treatment.

The serum level of cytokines detected in different groups (infected individual before and after treatment, controls from endemic and non-endemic area) were as follow. IL-5 in infected samples $9.41 \pm 12.49$ before, $13.99 \pm$ 29.90 after treatment. $3.79 \pm 8.27$ and $0.45 \pm 1.62$ endemic and non-endemic controls respectively. IL-10 in infected samples $32.22 \pm 53.84$ before and $7.47 \pm 11.85$ after treatment. $12.68 \pm 16.54$ and $10.01 \pm 18.18$ endemic and non-endemic controls respectively. The highest levels of the tested cytokines detected in the three groups was IL-10.

The multiple comparison of cytokines between different samples groups were summarized as follows. The comparison of IL-5 in infected samples with controls from endemic and in infected samples with none endemic controls was ( $\mathrm{P}=0.004$ and $\mathrm{P}=0.001$ respectively), but the comparison of IL-5 to the two controls showed no significant differences $(P=0.253)$. In case of IL-10 the comparison of infected samples with endemic controls was significant $(P=0.015)$, infected samples with non-endemic controls was also significant $(P=0.038)$, but the

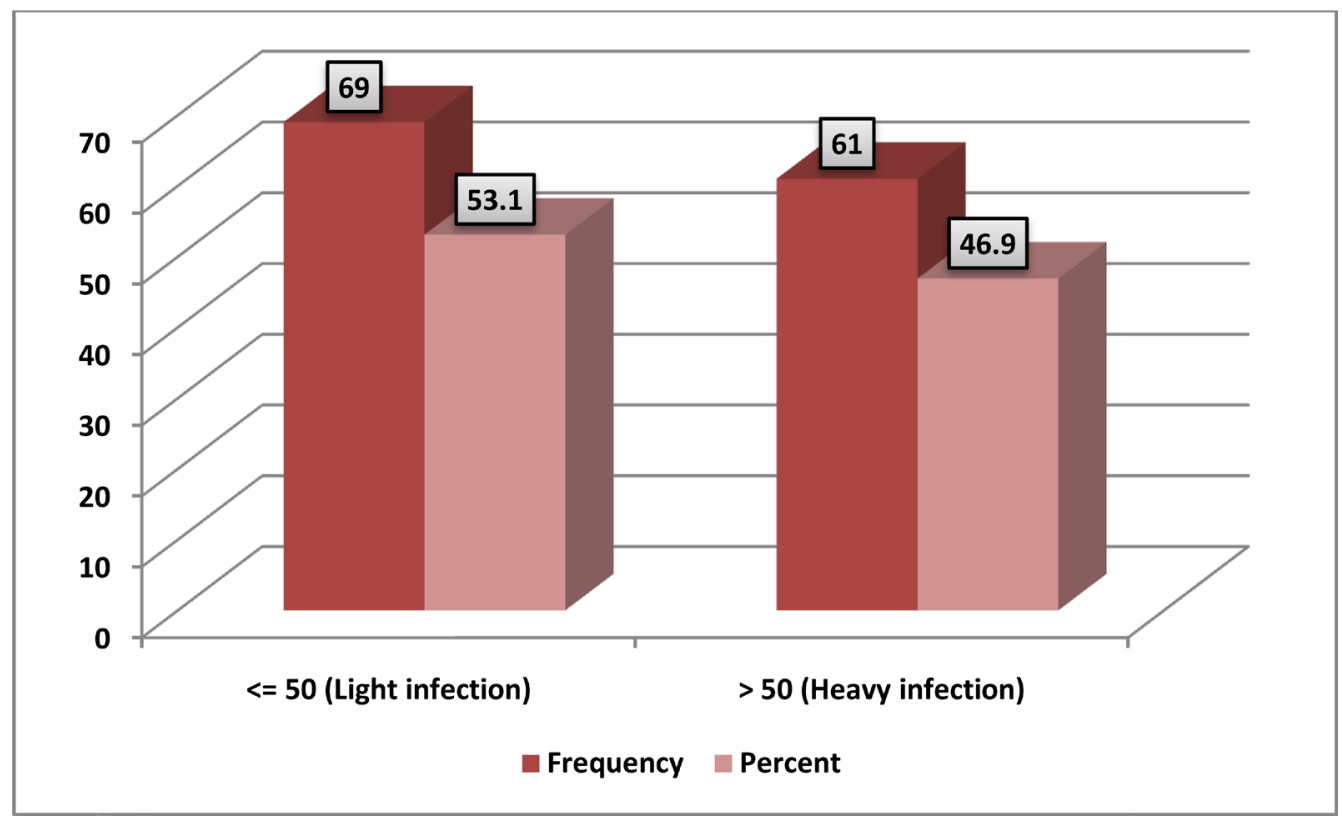

Figure 1. Frequency and percentages of the intensity of infection among Sudanese patients infected with Schistosoma haematobium. 


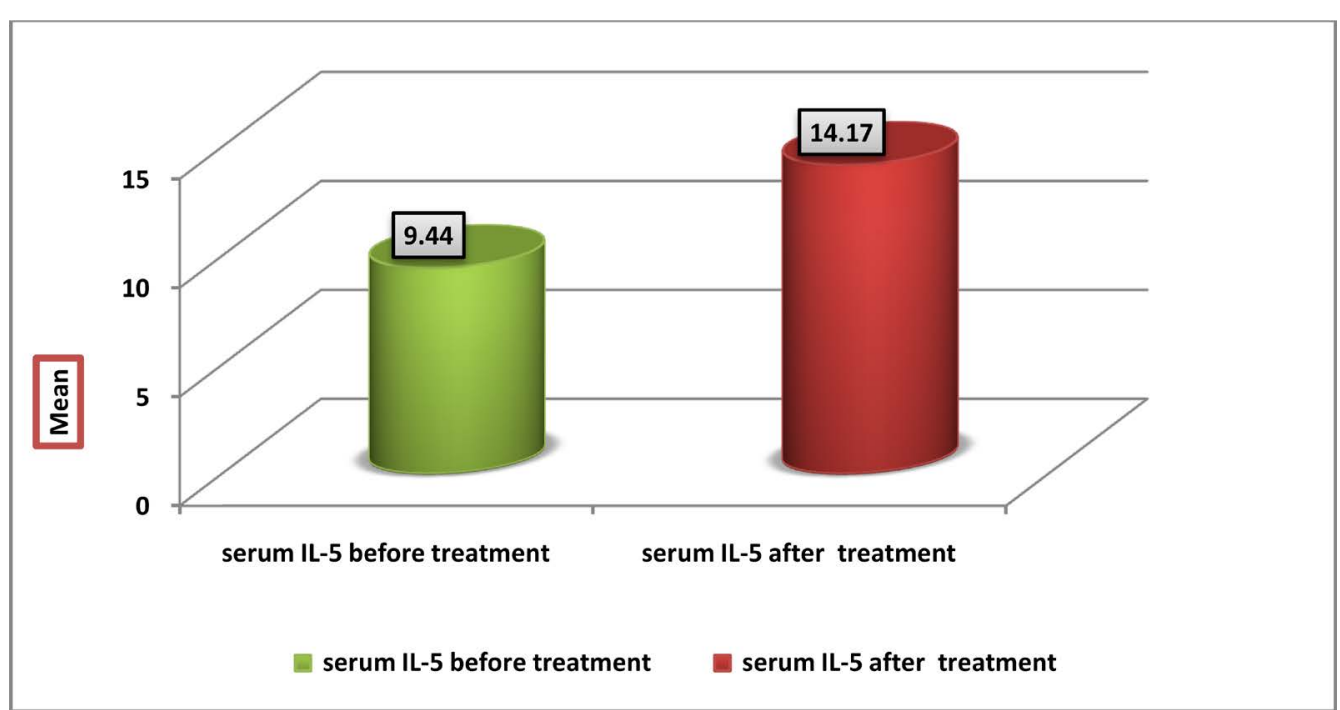

(a)

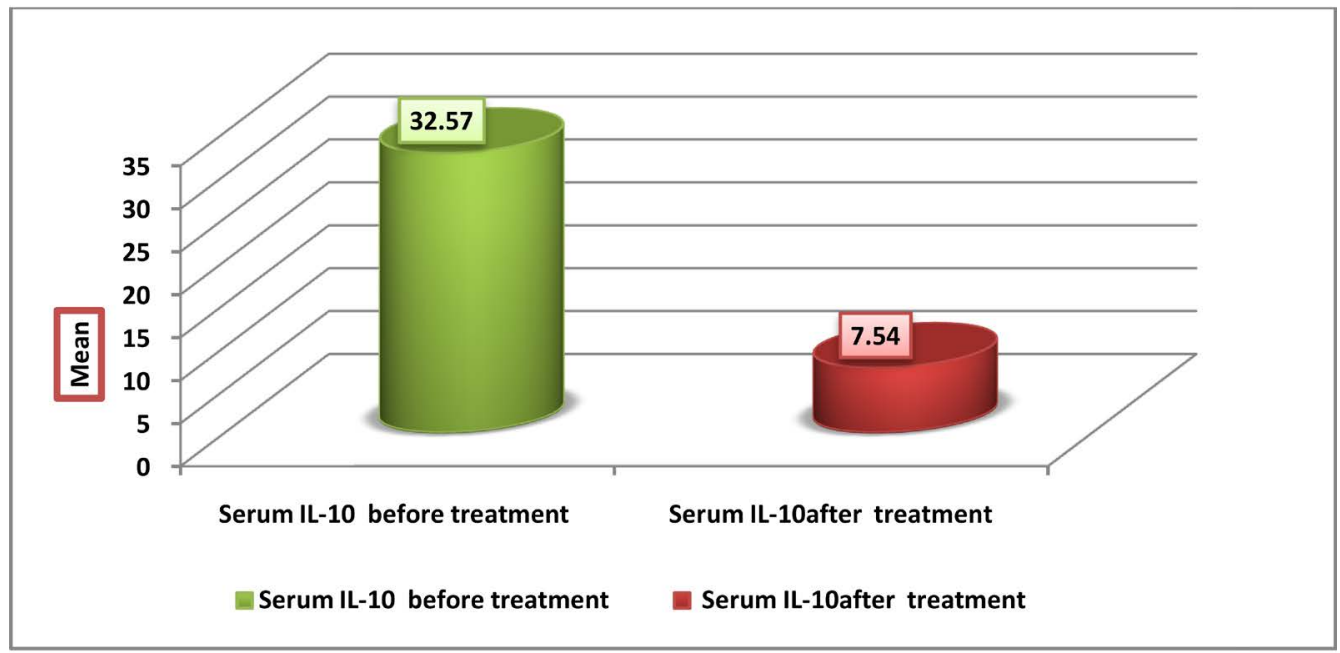

(b)

Figure 2. (a) Comparison of IL-5 levels in sera of Sudanese patients infected with Schistosoma haematobium before and after treatment; (b) Comparison of IL-10 levels in sera of Sudanese patients infected with Schistosoma haematobium before and after treatment.

Table 1. Cytokine profile in the urine of Sudanese patients infected with Schistosoma haematobium before and after treatment.

\begin{tabular}{cccccc}
\hline & Cytokine & Mean & N & Std. D. & P-value \\
\hline \multirow{2}{*}{ Pair 1 } & IL-5 in urine before treatment & 21.70 & 127 & 47.88 & 0.001 \\
& IL-5 in urine after treatment & 5.30 & 127 & 26.17 & \\
Pair 2 & IL-10 in urine before treatment & 3.37 & 124 & 4.33 & 0.477 \\
\hline
\end{tabular}

endemic controls with the non-endemic controls was not significant $(\mathrm{P}=0.824)$.

Table 2 shows sex cytokine profiles in patients before treatment. Females produce high levels of IL-10, than the males. And there was significant differences $(\mathrm{P}=0.0045)$. But there was no significant different in the production of IL-5 ( $\mathrm{P}=0.2888)$.

The age cytokine profiles before treatment were shown that. The IL-10 was significantly increased with age, but IL-5 did not follow age profile pattern. The differences in cytokines levels in all age groups were significant 
in case of IL-10. IL-5 shows no significant differences to age $(<13)$ vs $(13-23)(\mathrm{P}=0.0628$ and $\mathrm{P}=0.0749$ respectively).

Table 3 shows the relation between the intensity of the infection and cytokine production. All tested cytokines (IL-5 and IL-10) were found to be high in heavy infected individuals than in low infected. And the differences were statistically significant ( $\mathrm{p}=0.0143$, and 0.0381 respectively).

The levels of cytokines in urine according to the intensity of the infection were found to be as follows. IL-5 were high in heavy infection than in low infection, and IL-10 showed no change.

The correlation between cytokines levels and age in Schistosoma haematobium infection showed a positive correlation. IL-10 were strongly related to age $>23(\mathrm{r}=0.81 \mathrm{P}=0.0025)$, but IL-5 correlate with age $\leq 13(\mathrm{r}=$ $0.71 \mathrm{P}=0.0039)$.

\section{Discussion}

The relationship between age, intensity of infection and gender has been observed in many epidemiological situations, (endemic and epidemic) [20]-[22].

This study examined systemic cytokine responses in Sudanese individuals exposed to Schistosoma haematobium infection to determine the relationship between urinary and serum cytokine before and after treatment. The cytokines studied were anti-inflammatory, IL-5 as markers of Th2 responses, and IL-10, which originally classified as both Th1 and Th2 cytokine in humans [23]. Recently it considered as marker for immune-modulator responses including regulatory $\mathrm{T}$ cells responses [7] [24]. All participants had adetectable levels of all studied cytokines with different concentration. The Th2 like responses dominating later in response to infection, this shift in response from Th1 to Th2 has being enhanced by treatment [25]. The increase of IL-5 after treatment indicate an increase in anti-inflammatory, Th2, cytokine responses. This finding is similar to that reported by [11] and also this was supported by [26], who postulated that antigens released from dead worms would stimulate strong Th2 responses. It is therefore not surprising that pre-treatment cytokine levels in infected individual are low for the worm antigen that is believed to have immune evasion mechanism while in post-treatment the drugs would kill the worms and make the antigen available and become recognized by the immune response. IL-10 is an immunoregulatory cytokine that has a down-regulatory effect, particularly on Th1 responses in human, and mice [27] [28]. In human several studies have linked IgE responses and IL-5 with protection from reinfection [29]-[31]. Th1 responses have been linked to the so-called endemic normal [32] [33] such uninfected healthy individuals living in an area where Schist soma is transmitted produce higher levels of parasite specific IFN $\gamma$ and lower levels of IL-10. This coincide with our finding of these cytokine among endemic control individuals. This study also showed that there were high production of all tested cytokines in females than in males. Similar observation were seen in immunoglobulin's in other studies. We deduce these increases in female to hormonal function. Age cytokines profile in our study follow distinct age-profile with the relationship between age and cytokine except IL-5 which decreased by the aging. Normally levels of IL-10 response rose to peak early in childhood declining thereafter while levels of IL-5 rose more slowly to peak later. This discrepancy may be due

Table 2. Sex cytokine profile in serum before treatment.

\begin{tabular}{cccc}
\hline Cytokines & Sex & Mean + STD & T-test \\
\hline \multirow{2}{*}{ IL-5 } & Male $(\mathrm{n}=81)$ & $9.08 \pm 12.11$ & 5.84 \\
& Female $(\mathrm{n}=49)$ & $9.86 \pm 12.86$ & $0.2888^{\text {NS }}$ \\
IL-10 & Male $(\mathrm{n}=81)$ & $27.22 \pm 31.72$ & 28.77 \\
& Female $(\mathrm{n}=49)$ & $39.45 \pm 76.61$ & $0.0045^{*}$ \\
\hline
\end{tabular}

Table 3. Intensity of the infection and the cytokines levels in sera before treatment.

\begin{tabular}{cccc}
\hline Cytokines & Groups & Mean + SD & T-test \\
\hline \multirow{2}{*}{ IL-5 } & Light infected group $(\mathrm{n}=69)$ & $7.36 \pm 8.31$ & 11.33 \\
& Heavy infected group $(\mathrm{n}=61)$ & $11.66 \pm 15.50$ & $0.0143^{*}$ \\
IL-10 & Light infected group $(\mathrm{n}=69)$ & $30.73 \pm 60.52$ & 9.48 \\
& Heavy infected group $(\mathrm{n}=61)$ & $33.08 \pm 44.26$ & $0.0381^{*}$ \\
\hline
\end{tabular}


to the blocking or modulating effect of IL-10 on Th2 cytokine. So that the age groups where infection intensity and prevalence are lowest coincide with high IL-5 and light IL-10. Furthermore the study showed a positive correlation between age and all cytokines. So the IL-10 were increased with age while IL-5 decline with aging. This finding were comparable with [6]. In this study the intensity of the cytokines profile, showed high production of all tested cytokines in heavy infected individuals than light infected. IL-5 cytokines are believed to be important in protection against re-infection with Schistosoma haematobium [31] as well as IL-10 which has previously been associated with immunemodulation. The positive association between intensity of the infection and IL-5 were also reported by [34] in Brazilian Schistosoma mansoni infected individuals. Two possible explanation for this positive association are 1) IL-10 could simply be reflecting exposure to parasite antigens or 2) high levels of IL-10 are stimulated by high infection levels so as to prevent the development of excessive Th2-mediated pathology in addition to Th1-mediated pathology [35] [36]. The cytokine profile in urine showed almost similar results as serum, high before treatment and low after treatment. Some cytokines were detected more in urine than in sera this may be due to the possibility of local production of this cytokine in the bladder. These finding concur with those reported by [37] when studied cytokines in serum and urine of children infected with urinary bacteria. Very recently a similar finding was shown by [13] when studied urinary cytokine and related it with urinary tract pathology in children.

\section{Conclusion}

The low level of IL-5 that detected in study samples showed that the urinary Schistosomiasis in the study area could be considered in the acute stage of the disease. Also the result showed that IL-10 responses develop early compared to IL-5 responses and this definitely due to down modulating immune pathological responses that occur during the early phase of infection. IL-5 was working in reverse to IL-10 when studying these in sera and urine before and after treatment and we deduced that it was due to the modulating effect of IL-10. The study also showed that it is possible to use urine to study different cytokines in urinary Schistosomiasis which may indicate that there is a possibility of local production of cytokines in bladder.

\section{Acknowledgements}

We are grateful to the people of Hilat Salim and Saeed who agreed to participate in the study. A gratefully acknowledge to Abu Hania for his invaluable assistance in the field work. Financial support: This investigation received financial support from the Ministry of higher education and Scientific Research, special program for research and from the Faculty of Graduate Studies and Scientific Research National Ribat University.

\section{References}

[1] World Health Organization (2006) Schistosomiasis and Soil Transmitted Helminthinfedtions. Prelminary Estimates of the Number of Children Treated with Albendazole or Mebendazole.

[2] Chitsulo, L., Engels, D., Montresor, A.S. and Savioli, L. (2000) The Global Status of Schistosomiasis, and It Is Control. Acta Tropica, 77, 41-51. http://dx.doi.org/10.1016/S0001-706X(00)00122-4

[3] Mabata, T., Ovji, M. and Oguoma, V.M. (2009) The Prevalence of Urinary Schistosomiasis in Ogbadibo Local Government Area of Benue State, Nigeria. The Journal of Infectious Diseases, 98.

[4] Rollinson, D. (2009) A Wake up Call for Urinary Schistosomiasis: Reconciling Research Effort with Public Health Importance. Parasitology, 136, 1593-1610. http://dx.doi.org/10.1017/s0031182009990552

[5] Butterworth, A.G. (1998) Immunology Aspects of Schistosomiasis. British Medical Bulletin, 54, 357-368. http://dx.doi.org/10.1093/oxfordjournals.bmb.a011693

[6] Milner, T., Reilly, L., Nausch, N., Midlzi, N., Mduluza, T., Maizels, R. and Mutapi, F. (2010) Circulating Cytokine Levels and Antibody Responses to Human Schistosoma haematobium: IL-5 and IL-10 Levels Depend upon Age and Infection Status. Parasite Immunology, 32, 710-721. http://dx.doi.org/10.1111/j.1365-3024.2010.01235.x

[7] Hesse, M., Piccirillo, A.C., Belkaid, Y., Prufer, J., Mentink-Kane, M., Leusink, M., Cheever, A.W., Shevach, E.M. and Wynn, T.A. (2004) The Pathogensis of Schistosomiasis Is Controlled by Cooperating IL-10-Producing Innate Effector and Regulatory T Cells. Journal of Immunology, 172, 3157-3166. http://dx.doi.org/10.4049/jimmunol.172.5.3157

[8] Mutapi, F., Winborn, G., Midzi, N., Taylor, M., Mduluza, T. and Maizel, R.M. ( 2007) Cytokine Responses to Schistosoma haematobium in a Zimbawean Population: Contrasting Profiles for IFN- $\alpha$, IL-4, IL-5 \& IL-10 with Age. BMC Infectious Diseases, 7, 139. http://dx.doi.org/10.1186/1471-2334-7-139 
[9] Scott, J.T., Turner, C.M.R., Mutapi, F., Woolhouse, M.E.J., Ndhlovu, P.D. and Hagan, P. (2001) Cytokine Responses to Mitogen and Schistosoma haematobium Antigens Are Different in Children with Distinct Infection Histories. Parasite Immunology, 23, 519-526. http://dx.doi.org/10.1046/j.1365-3024.2001.00409.x

[10] Scott, J.T., Turner, C.M.R., Mutapi, F., Woolhouse, M.E.J., Chandiwana, S.K., Mduluza, T., Ndhlovu, P.D. and Hagan, P. (2000) Dissociation of Interleukin-4 and Interleukin-5 Production Following Treatment for Schistosoma haematobium Infection in Human. Parasite Immunology, 22, 341-348. http://dx.doi.org/10.1046/j.1365-3024.2000.00311.x

[11] Van den Biggelaar, A.H.J. and Borrmann, S. (2002) Immune Responses Induced by Repeated Treatment Do Not Result in Protective Immunity to Schistosoma haematobium: Interleukin-5 and IL-10 Responses. Journal of Infectious Diseases, 186, 1474-1482.

[12] Agace, W., Hedges, S., Andersson, U., Andersson, J., Ceska, M. and Svanborg, C. (1993) Selective Cytokine Production by Epithelial Cells Following Exposure to Escherichia coli. Infection and Immunity, 61, 602-609.

[13] Njaanake, K.H., Simonsen, P.E., Vennervald, B.J., Mukoko, D.A., et al. (2014) Urinary Cytokines in Schistosoma haematobium-Infected Schoolchildren from Tana Delta District of Kenya. BMC Infectious Diseases, 14, 501. http://dx.doi.org/10.1186/1471-2334-14-501

[14] Cheesbrough, M. (1998) District Laboratory Practice in Tropical Countries. Part 1. Cambridge University Press, London.

[15] Peter, P. Mahmoud, A. Warren, K. Ouma, J. and Siogok, T. (1976) Field Studies of a Rapid Accurate Means of Quantifying Schistosoma haematobium Eggs in Urine Samples. Bulletin of the World Health Organization, 54, $159-162$.

[16] World Health Organization (1998) Guidelines for the Evaluation of Soil-Transmitted Helminthiasis and Schistosomiasis at Community Level. World Health Organization, Geneva.

[17] Bergquist, R., Johansen, M.V. and Utzinger, J. (2009) Diagnostic Dilemmas in Helminthology: What Tools to Use and When? Trends in Parasitology, 25. http://dx.doi.org/10.1016/j.pt.2009.01.004

[18] Katz, N., Coelho, P.M. and Pellegrino, J. (1972) Evaluation of Katos Quantitative Method through the Recovery of Schistosoma mansoni Eggs Added to Human Feces. The Journal of Parasitology, 56, 1032-1033. http://dx.doi.org/10.2307/3277532

[19] Sokal, R.R. and Biometry, R.J. (1995) The Principles and Practice of Statistics in Biological Research. Freeman and Company.

[20] Hagan, P., Ndhlovu, P.D. and Dunn, D. (1998) Schistosoma Immunology: More Question than Answers. Parasitology Today, 14, 407-412. http://dx.doi.org/10.1016/S0169-4758(98)01325-8

[21] Stelma, F.F., Talla, I., Polman, K., Niang, M., Sturrock, R.F., Deelder, A.M. and Gryseels, B. (1993) Epidemiology of Schistosoma mansoni Infection in a Recently Exposed Community in Northern Senegal. The American Journal of Tropical Medicine and Hygiene, 49, 701-706.

[22] van Dam, G.J., Stelma, F.F., Gryseels, B., Falcão Ferreira, S.T.M., Talla, I., Niang, M., Rotmans, J.P. and Deelder, A.M. (1996) Antibody Response Patterns against Schistosoma mansoni in a Recently Exposed Community in Senegal. The Journal of Infectious Diseases, 173, 1232-1241. http://dx.doi.org/10.1093/infdis/173.5.1232

[23] Del Prete, G., De Carli, M., Almerigogna, F., Giudizi, M.G., Biagiotti, R. and Romagnani, S. (1993) Human IL-10 Is Produced by Both Type 1 Helper (Th1) and Type 2 Helper (Th2) T Cell Clones and Inhibits Their Antigen-Specific Proliferation and Cytokine Production. The Journal of Immunology, 150, 353-360.

[24] McKee, A.S. and Pearce, E.J. (2004) CD25 ${ }^{+}$CD4 ${ }^{+}$Cells Contribute to Th2 Polarization during Helminthes Infection by Suppressing Th1 Response Development. The Journal of Immunology, 173, 1224-1231. http://dx.doi.org/10.4049/jimmunol.173.2.1224

[25] De Souza, J.R., Morais, C.N.L., et al. (2007) Treatment of Human Acute Schistosomiasis with Oxamniquine Induces an Increase in Interferon- $\gamma$ Response to Schistosoma mansoni Antigens. Memórias do Instituto Oswaldo Cruz, 102, 225-228. http://dx.doi.org/10.1590/S0074-02762007005000002

[26] Woolhouse, M.E.J. and Chandiwana, S.K. (1990) The Epidemiology of Schistosome Infections of Snails: Taking the Theory into the Field. Parasitology Today, 6, 65-70.

[27] Hoffmann, K.F., James, S.L., Cheever, A.W. and Wynn, T.A. (1999) Studies with Double Cytokine-Deficient Mice Reveal That Highly Polarized Th1- and Th2-Type Cytokine and Antibody Responses Contribute Equally to Vaccine-Induced Immunity to Schistosoma mansoni. The Journal of Immunology, 163, 927-938.

[28] Hoffmann, K.F., Cheever, A.W. and Wynn, T.A. (2000) IL-10 and the Dangers of Immune Polarization: Excessive Type 1 and Type 2 Cytokine Responses Induce Distinct Forms of Lethal Immunopathology in Murine Schistosomiasis. The Journal of Immunology, 164, 6406-6416. http://dx.doi.org/10.4049/jimmunol.164.12.6406

[29] Hagan, P., Blumenthal, U.J., Dunn, D., Simpson, A.J. and Wilkins, H.A. (1991) IgE, IgG4 and Resistance to reinfection with Schistosoma haematobium. Nature, 349, 243-245. http://dx.doi.org/10.1038/349243a0 
[30] Grogan, J.L., Kremsner, P.G., Deelder, A.M. and Yazdanbakhsh, M. (1998) Antigen-Specific Proliferation and Interferon- $\gamma$ and Interleukin-5 Production are Down-Regulated during Schistosoma haematobium Infection. The Journal of Infectious Diseases, 177, 1433-1437. http://dx.doi.org/10.1086/517832

[31] Medhat, A., Shehata, M., Buccci, K., et al. (1998) Increased Interleukin-4 and Interleukin-5 Production in Response to Schistosoma haematobium Adult Worm Antigens Correlates with Lack of Reinfection after Treatment. The Journal of Infectious Diseases, 178, 512-519. http://dx.doi.org/10.1086/515630

[32] Gazzinelli, G., Viana, I.R., Bahia-Oliveira, L.M., et al. (1992) Immunological Profiles of Patient from Endemic Areas Infected with Schistosoma mansoni. Memórias do Instituto Oswaldo Cruz, 87, 139-142.

[33] Viana, I.R., Sher, A., Carvalho, O.S., et al. (1994) Interferon- $\gamma$ Production by Peripheral Blood Mononuclear Cell from Residents of an Area Endemic for Schistosoma mansoni. Transactions of the Royal Society of Tropical Medicine and Hygiene, 88, 466-470.

[34] Silveira, A.M., Gazzinelli, G., Alves-Oliveira, et al. (2004) Human Schistosomiasis mansoni: Intensity of Infection Differentially Affects the Production of Interleukin-10, Interferon- $\gamma$ and Interleukin-13 by Soluble Egg Antigen or Adult Worm Antigen Stimulated Cultures. Transactions of the Royal Society of Tropical Medicine and Hygiene, 98, 514-519. http://dx.doi.org/10.1016/j.trstmh.2003.11.009

[35] Pearce, E.J. and MacDonald, A.S. (2002) The Immunobiology of Schistosomiasis. Nature Reviews Immunology, 2, 499-511. http://dx.doi.org/10.1038/nri843

[36] Taylor, J.J., Mohrs, M. and Peare, E.J. (2006) Regulatory T Cell Responses Develop in Parallel to Th Responses and Control the Magnitude and Phenotype of the Th Effector Populatio. The Journal of Immunology, 176, 5839-5847. http://dx.doi.org/10.4049/jimmunol.176.10.5839

[37] Gurgoze, M.K., Akarsu, S., Yilmaz, E., Gödekmerdan, A., Akça, Z., Ciftçi, I. and Aygün, A.D. (2005) Proinflammatory Cytokines and Procalcitonin in Children with Acute Pyelonephritis. Pediatric Nephrology, 20, 1445-1448. 